\title{
Influência da Personalidade Tipo D na Qualidade de Vida da Doença Inflamatória Intestinal
}

\author{
Influence of Type D Personality in Quality of Life of Inflammatory Bowel Disease
}

\author{
Rute Amorim ${ }^{1}$ e Marina Prista Guerra ${ }^{2}$
}

\begin{abstract}
Resumo
Realizaram-se dois estudos com uma amostra de 208 participantes, pertencentes a uma Associação, com Doença Inflamatória Intestinal: 61\% com Doença de Crohn e 39\% com Colite Ulcerosa, maioritariamente mulheres $(63,9 \%)$ e com média de idade 36,7 anos. Avaliaram-se as variáveis independentes em estudo: ansiedade, depressão, stress percebido, alexitimia e personalidade tipo D. A escala de personalidade não estava adaptada para português; no estudo 1, procedeu-se à replicação das análises psicométricas do estudo original. No estudo 2 determinou-se a predição das duas componentes da variável dependente "qualidade de vida". Através de regressões múltiplas hierárquicas, os resultados indicaram que, controlando a alexitimia, depressão e ansiedade são as variáveis com maior contribuição na predição da componente física. Na componente mental, controlando alexitimia e personalidade tipo D, observou-se que a depressão, ansiedade, perceção de stress e idade são preditores significativos. A personalidade tipo D tem maior prevalência nesta patologia do que em populações saudáveis.
\end{abstract}

Palavras-chave: doença inflamatória intestinal, qualidade de vida, personalidade tipo D, alexitimia

\begin{abstract}
Two studies were performed with a sample of 208 participants, belonging to an Association, with Inflammatory Bowel Disease: 61\% with Crohn's Disease and 39\% Ulcerative Colitis, mostly women $(63,9 \%)$ and with 36,7 age mean. The independent variables in studies were evaluated: anxiety, depression, perceived stress, alexithymia and type D personality. The personality scale was not adapted to Portuguese; in study 1, psychometric analyses were performed according to the original study. In study 2 the prediction of the two components of the dependent variable "quality of life" was performed. Through multiple hierarchical regressions, the results have shown that, controlling alexithymia, depression and anxiety are the variables with the highest contribution in predicting the physical component. In the mental component, controlling alexithymia and type D personality, it's observed that depression, anxiety, perceived stress and age are significant predictors. Type D personality has a higher prevalence in this pathology than in healthy populations.
\end{abstract}

Keywords: inflammatory bowel disease, quality of life, type D personality, alexithymia

Agradecimento: Este trabalho foi financiado por Fundos Nacionais através da FCT - Fundação para a Ciência e a Tecnologia no âmbito do projecto PEst-OE/PSI/UI0050/2014. Agradecemos ainda a colaboração da Associação Portuguesa de Doentes com Doença Inflamatória Intestinal e em especial ao Dr. Jorge Ascenção e Dra. Vera Coelho.

\footnotetext{
${ }^{1}$ Mestre em Psicologia Clínica e da Saúde. Faculdade de Psicologia e de Ciências da Educação da Universidade do Porto. Rua Alfredo Allen, 4200-135 Porto, Portugal. Tel: +351 226079700. E-mail: mipsi09022@fpce.up.pt

2 Professora Associada com Agregação. Faculdade de Psicologia e de Ciências da Educação da Universidade do Porto. Rua Alfredo Allen, 4200-135 Porto, Portugal. Tel: +351 226079700. E-mail: mguerra@ fpce.up.pt
} 


\section{Introdução}

O termo Doença Inflamatória Intestinal (DII) é utilizado para referir pelo menos duas condições crónicas, sem um curso clínico previsível e com momentos de remissão e momentos de exacerbação/recaída, que, embora apresentem aspetos clínicos e terapêuticos comuns (sendo esta semelhança que as engloba na mesma categoria), são distintas: Colite Ulcerosa (CU) e Doença de Crohn (DC) (Zhou, Ren, Irvine, \& Yang, 2010).

A principal diferença entre as duas doenças é o facto de a DC ser uma inflamação que pode afetar toda a parede do intestino e pode envolver qualquer parte do sistema gastrointestinal (desde a boca até ao ânus), e da CU se limitar usualmente à mucosa e afetar quase exclusivamente o cólon (Nasseri-Moghaddam, 2012).

Embora possamos enunciar sintomas particulares de acordo com cada uma das doenças, os sintomas mais globais da DII são diarreia, dor abdominal, fadiga e cansaço. Contudo, consequências associadas à DII, como artrite, fístulas, abcessos e problemas de pele também podem contribuir para uma qualidade de vida mais pobre. Apesar da origem da DII não ser conhecida, acredita-se que fatores genéticos, imunitários e ambientais interajam juntos. Isto é, se já existe uma predisposição genética, certos fatores ambientais e psicológicos podem influenciar as defesas imunitárias e desencadear os sintomas (Sajadinejad, Asgari, Molavi, Kalantari, \& Adibi, 2012a).

A DII, embora possa acontecer em qualquer idade, ocorre mais frequentemente entre os $15 \mathrm{e}$ os 30 anos. Além disso, tem-se verificado que, apesar de estar a aumentar noutras partes do mundo, é mais comum no mundo industrializado e no oeste; é também mais comum em classes socioeconómicas elevadas e áreas urbanas (Nasseri-Moghaddam, 2012).

O principal objetivo ao tratar a DII é induzir e manter a remissão pelo máximo de tempo possível. Para tal, podem utilizar-se uma vasta gama de medicamentos, podendo haver necessidade de adaptações alimentares e havendo, ainda, a hipótese de optar por medicinas alternativas/complementares. Além disso, é frequente que estes indivíduos necessitem de cirurgia; estima-se que $80 \%$ dos pacientes com
DC precisarão de algum tipo de cirurgia e $25 \%$ dos pacientes com CU de uma colostomia (Todorovic, 2012).

Hoje em dia, torna-se cada vez mais reconhecida a importância de uma abordagem multifatorial que tenha em conta as várias facetas que compõem a vida de um indivíduo (Salas \& Garzón, 2013). Assim, acreditando-se na influência de fatores de diferentes origens na evolução e comportamento da doença é importante considerar as influências psicológicas.

Estas caraterísticas psicológicas podem revelar-se em aspetos como a adesão aos regimes de tratamento, a atualização do conhecimento sobre a doença e tratamentos disponíveis ou a gestão dos sintomas e da dor (Todorovic, 2012). Além disso, podem influenciar a qualidade de vida dos indivíduos que têm lidar com as exigências desta doença crónica.

\section{Qualidade de vida na DII}

No campo de investigação da saúde/doença uma das dimensões da qualidade de vida (QV) mais estudada é a qualidade de vida relacionada com a saúde (QVRS), sendo que os dois conceitos são frequentemente utilizados como sinónimos na literatura desta área.

A revisão de Sainsbury e Heatley (2005) indica algumas tendências de fatores não psicológicos que, em indivíduos com DII, influenciam de modo negativo a QV. Encontramse entre elas: a) ser do sexo feminino, b) ter um nível socioeconómico mais baixo; c) ter um nível educacional mais baixo; d) o aumento da idade; e) fumar. Por seu lado, Zhou et al. (2010) não encontram diferenças no que diz respeito à influência do género, idade ou nível educacional. A QV encontra-se debilitada em indivíduos com DII quando comparados com a população saudável (Zhou et al., 2010; Nordin, Pahlman, Larsson, Sundberg-Hjelm, \& Lööf, 2002; IglesiasRey et al., 2014). No entanto, a QV é melhor nos indivíduos em remissão do que naqueles que se encontram num momento de estado ativo da doença, o que demonstra a importância do tratamento da DII ainda que continuem a existir impactos mesmo numa fase em que a doença se encontra controlada (Zhou et al., 2010).

No que diz respeito a diferenças entre as duas doenças agrupadas na designação DII, os 
resultados são contraditórios. Enquanto o estudo de Nordin et al. (2002) apresenta resultados que indicam que o grupo de indivíduos com DC tem uma qualidade de vida pior, o que parece ser uma tendência da maioria dos estudos que referem diferenças (Meder, Swiatkowaski, Meder, \& Koza, 2010), o estudo de Zhou et al. (2010) não encontra diferenças estatisticamente significativas entre as duas doenças. De facto, os últimos mencionam estudos nos quais as diferenças na $\mathrm{QV}$ na CU e DC deixam de ser significativas após o ajustamento das diferenças de severidade da doença.

São vários os fatores descritos na literatura como podendo influenciar a $\mathrm{QV}$, concluindo-se que um nível elevado dessas variáveis se encontra relacionado com baixa QV. Entre esses fatores encontram-se, como poderá ser comprovado ao longo deste enquadramento: alexitimia, ansiedade, depressão, personalidade tipo $\mathrm{D}$ e stress percebido.

O presente estudo pretende aumentar a informação disponível relativamente ao impacto dos referidos factores, ao mesmo tempo que salienta a importância de avaliar nesta doença um constructo recente e pouco estudado: a personalidade tipo D.

\section{Alexitimia}

A alexitimia apresenta correlações com variáveis sociodemográficas estando mais presente: a) com o avançar da idade, b) no sexo masculino, c) em indivíduos com um estatuto socioeconómico baixo, d) em indivíduos com escolaridade mais baixa, e) no estado civil de solteiro, e f) em indivíduos com pior perceção da saúde (Lane, Sechrest, \& Riedel, 1998; Mattila, Saarni, Salminen, Huhtala, Sintonen, \& Joukamaa, 2009).

O estudo de Ben Thabet et al. (2012) reporta $54 \%$ da amostra de indivíduos com DII como tendo valores de alexitimia - um valor significativamente superior ao do grupo de controlo. Embora esta não seja uma conclusão consensual é tendência em alguns estudos, apresentando-se a alexitimia um traço de personalidade comum nos indivíduos com DII; também tem sido verificada uma relação próxima da alexitimia com a ansiedade e a depressão (Iglesias-Rey et al., 2012; Mattila et al., 2009).
Ainda que a alexitimia não seja específica de doentes com DII pode, no entanto, fazer com que os pacientes traduzam o sofrimento psicológico através de sintomas somáticos em vez de o fazerem pela comunicação verbal (Sajadinejad et al., 2012a). De facto, o estudo de Lunazzi de Jubany (2000) demonstra diferenças entre uma população normativa e uma psicossomática, sendo a alexitimia superior na segunda. Já numa amostra clínica composta por indivíduos com DII, o estudo de Branco (2008) demonstra uma prevalência significativa de $32,6 \%$ de sujeitos alexitimicos, quando em comparação com os $13 \%$ da amostra de controlo.

Vários estudos concluem que a alexitimia faz parte dos determinantes da QV nesta população clínica (Boye et al., 2008; Iglesias-Rey et al., 2012; Mattila et al., 2009), estando relacionada com uma perceção mais debilitada da saúde e qualidade de vida e com menor satisfação com a vida (Boye et al., 2008).

No que diz respeito à distinção entre $\mathrm{CU}$ e DC, Iglesias-Rey et al. (2012) não encontram diferenças no tipo de doença ou na questão da recaída (isto é, níveis mais elevados de alexitimia aquando de um momento de exacerbação da doença). Já Boye et al. (2008) concluem que na $\mathrm{CU}$ o impacto negativo da alexitimia se revela mais quando a doença está num momento de atividade alta e que na DC há uma relação, ainda que sem explicação, de proporcionalidade direta entre os valores da alexitimia e limitações no desempenho físico.

\section{Ansiedade e depressão}

A ansiedade e a depressão, embora aqui apresentados em conjunto $\mathrm{e}$ frequentemente estudados em simultâneo, são constructos diferentes. Ainda que seja comum encontrar ambas as perturbações associadas há distinção entre elas (Anxiety and Depression Association of America, 2010-2014).

A ansiedade e a depressão têm maior prevalência na população com DII do que na saudável. Goodhand et al. (2012), comparando uma amostra controlo saudável com uma amostra de pacientes com DII, concluem que os valores de ansiedade e depressão dos segundos são o dobro dos valores dos primeiros. Ainda que em vários estudos os valores de prevalência da ansiedade e 
da depressão na população com DII não sejam consensuais é possível concluir que numa fase ativa da doença se encontram valores de prevalência mais elevados do que numa fase de remissão (Sajadinejad et al., 2012a; Nahon et al., 2012; Meder et al., 2010; Ben Thabet et al., 2012). Embora os resultados de Meder et al. (2010) e Goodhand et al. (2012) não refiram diferenças na prevalência entre indivíduos com DC e CU, Goodhand et al. (2012) refere alguns fatores que afetam mais particularmente cada doença e Nordin et al. (2002) refere que os níveis são mais elevados nos primeiros em comparação com os segundos - resultados que desaparecem após o ajuste da severidade dos sintomas físicos. Assim, os resultados indicam uma relação estreita entre sofrimento psicológico e físico, sendo de notar que a ansiedade e a depressão parecem estimular a produção de citoquinas proinflamatórias que pioram o curso da DII (Sajadinejad et al., 2012a). Além disso, Goodhand et al. (2012) referem a existência de outros fatores que afetam a depressão e ansiedade sendo de realçar o stress percebido.

A relação entre níveis elevados de ansiedade e depressão e níveis mais baixos de QV nos indivíduos com DII é comprovada por vários estudos (Iglesias-Rey et al., 2014; Nordin et al., 2002; Hauser, Tkalcić, Stimac, Milić, \& Sincić, 2011; Faust, Halpern, Danoff-Burg, \& Cross, 2012).

Os resultados de Hauser et al. (2011) referem, indo ao encontro do que é referido na literatura, que a qualidade de vida nas mulheres da sua amostra é menor do que a dos homens. Uma das explicações apresentadas pelos investigadores é o facto de as mulheres revelarem um nível significativamente mais elevado de depressão e ansiedade, revelando ter a sua QV mais afetada. Os investigadores referem, ainda, que pode ser a influência da ansiedade e depressão na perceção dos sintomas físicos a contribuir para este relato mais negativo quanto à $\mathrm{QV}$.

\section{Perceção do stress}

Sainsburry e Heatley (2005) reportam-se a duas revisões que concluem que os sintomas da DII estão mais relacionados com o stress diário ou crónico do que eventos de vida major. Apesar de Kuroki et al. (2011) também referirem que vários estudos indicam a possível existência de uma correlação entre a perceção de stress e a atividade da DII, o estudo destes investigadores não encontrou diferenças significativas no nível de stress percebido entre os indivíduos do grupo de controlo e os do grupo com DII.

Ainda que a DII já não seja considerada uma doença psicossomática verificam-se frequentemente relatos de exacerbação por influência do stress (Moradkhani et al., 2013). Sugrue, Landers, McCarthy e Buckley (2013) indicam que os participantes que reportam um nível mais elevado de stress percebido têm níveis mais elevados na sua perceção da atividade da doença, sendo que os resultados de Iglesias et al. (2011) remetem para uma perceção mais elevada em indivíduos que não estão em remissão. Além disso, os mesmos autores não encontram diferenças entre a perceção dos indivíduos com CD ou UC, encontrando, no entanto, diferenças relativamente ao sexo: nas mulheres a perceção do stress é mais elevada do que nos homens.

Tudoran e Furtunescu (2013), avaliando o stress percebido numa amostra de adultos romenos saudáveis, verificam um ciclo de influência entre esta variável e a QV: a perceção mais elevada do stress relaciona-se com uma perceção mais baixa da $\mathrm{QV}$; a perceção negativa da QV aumenta o stress. Verifica-se igualmente na DII que níveis elevados de stress percebido afetam de modo negativo a QV (Moradkhani et al., 2013; Iglesias-Rey et al., 2014).

\section{Personalidade tipo D ou 'distressed personality'}

Apesar de ainda não ter sido possível integrar aspetos como os até aqui mencionados num tipo de personalidade (Sajadinejad, Molavi, Asgari, Kalantari, \& Adibi, 2012b), existe um que tem sido um alvo recente de investigação em várias doenças crónicas: a personalidade tipo D (ou 'distressed personality').

Este construto, apresentado por Denollet em 1996, encontra-se associado a valores altos de inibição social e afetividade negativa. A inibição social relaciona-se com uma maior tendência em não demonstrar emoções e comportamentos quando em situações sociais, nomeadamente em que o indivíduo perceciona a expressão emocional como possível alvo de julgamento negativo. Além disso, a afetividade negativa é um traço que se 
relaciona com a vivência de estados emocionais adversos (Denollet, 2012).

Quando em comparação com indivíduos sem este tipo de personalidade, indivíduos com personalidade tipo D apresentam uma QV afetada (Sararoudi, Afshar, Adibi, Daghaghzadeh, Fallah, \& Abotalebian, 2011; Hansel, Umar, Lunsford, Harris, Dibaise, \& Crowell, 2010). Associam-se à personalidade tipo D níveis mais elevados de stress percebido (Polman, Borkoles, \& Nicholls, 2010) e maior propensão a fatores como depressão e ansiedade (Denollet, 2005). Os resultados de Williams, Curren e Bruce (2011) demonstram que a personalidade tipo $\mathrm{D}$ e a alexitimia têm uma correlação positiva e significativa; além disso, apesar de uma certa sobreposição conceptual são construtos diferentes.

A investigação existente quanto a esta variável e a sua relação com a DII, embora não extensa, apresenta alguns aspetos importantes.

Hansel et al. (2010), na sua investigação com pacientes com Desordens Gastrointestinais Funcionais, que incluem a DII, encontram valores que demonstram que $37 \%$ da sua amostra apresenta personalidade tipo D. A investigação de Sajadinejad et al. (2012b), comparando uma amostra de mulheres com CU com o grupo controlo, refere a prevalência da personalidade tipo D como de $59 \%$ e $33 \%$ respetivamente. Contudo, uma vez que o estudo não realiza comparação com dados relativos à prevalência da personalidade tipo D na DC nem inclui o sexo masculino, não é possível precisar se existem diferenças entre as duas doenças ou se os valores são comuns aos dois sexos.

Também a investigação do impacto deste tipo de personalidade na QV em indivíduos com DII é demonstrada. Sararoudi et al. (2011) encontram uma relação significativa da componente da afetividade negativa com a $\mathrm{QV}$ e, embora não significativa, uma tendência da componente inibição social. Além disso, a presença de traços de personalidade como os relacionados com a personalidade tipo $\mathrm{D}$ podem aumentar a perceção da severidade dos sintomas e ter um impacto negativo na perceção da QV (Hansel et al. 2010). Também a investigação de Sajadinejad et al. (2012b) mostra que a parte da sua amostra com personalidade tipo $\mathrm{D}$ tem valores de $\mathrm{QV}$ mais baixos.

\section{Objetivos}

Este estudo apresenta como variáveis dependentes a Componente Medida Sumária Física (CMSF) e a Componente Medida Sumária Mental (CMSM) - que, em conjunto, retratam a QV - e como variáveis independentes a alexitimia, a ansiedade, a depressão, o stress percebido e a personalidade tipo D. Uma vez que a escala da Personalidade tipo $\mathrm{D}$ não estava avaliada psicometricamente para o Português, após solicitação de autorização ao autor Denolett, foi objetivo reproduzir a avaliação psicométrica original em portugueses com DII no Estudo 1. Após a realização da revisão bibliográfica estabelecem-se como objetivos para a investigação do Estudo 2:

a) Caraterizar a amostra ao nível das variáveis sociodemográficas e clínicas; dependentes e independentes;

b) Verificar a prevalência da personalidade tipo D na DII;

c) Verificar a relação das variáveis independentes com as variáveis dependentes;

d) Verificar a influência do tipo de doença nas variáveis em estudo;

e) Estabelecer um modelo preditor para cada uma das variáveis dependentes.

A presente investigação compreende assim dois estudos: Estudo 1 (adaptação da escala de Personalidade tipo $\mathrm{D}$ para uma população portuguesa com DII) e Estudo 2.

\section{Metodologia}

\section{Participantes}

A amostra do presente estudo (Estudo $1 \mathrm{e}$ Estudo 2) é constituída por 208 participantes, tendo sido determinados como critérios de inclusão: a) Ter idade igual ou superior a 18 anos; b) Ter um diagnóstico de Doença Inflamatória Intestinal; c) Ser residente em Portugal.

Através da análise da amostra (Quadro 1) verifica-se que existe prevalência de participantes do sexo feminino e que as idades dos participantes variam desde os jovens aos idosos. No que diz respeito ao estado civil, destacam-se os participantes casados e solteiros e, relativamente à escolaridade, os participantes com ensino universitário. A amostra é constituída por um número mais elevado de participantes com 
Quadro 1. Frequências relativas à amostra em estudo

\begin{tabular}{lccccc}
\hline & $\mathrm{N}$ & $\%$ & $\mathrm{M}$ & $\mathrm{DP}$ & Amplitude \\
\hline Sexo & & & & & \\
$\quad$ Feminino & 133 & 63.9 & & & \\
$\quad$ Masculino & 75 & 36.1 & & & $18-72$ \\
Idade & & & 36.69 & 10.01 & \\
Estado Civil & 94 & 45.2 & & & \\
$\quad$ Solteiro/a & 104 & 50 & & & \\
$\quad$ Casado/a & 9 & 4.3 & & & \\
$\quad$ Divorciado/a & 1 & 0.5 & & & \\
$\quad$ Viúvo/a & & & & & \\
Grau de escolaridade & 6 & 2.9 & & & \\
$\quad$ Ensino Básico & 72 & 34.6 & & & \\
$\quad$ Ensino Secundário & 130 & 62.5 & & & \\
$\quad$ Ensino Superior & 126 & 60.6 & & & \\
Diagnóstico & 82 & 39.4 & & & \\
$\quad$ Doença de Crohn & & & 112.9 & 95.30 & $2-552$ \\
$\quad$ Colite Ulcerosa & & & 0 & & \\
Tempo desde início de & & & 99.78 & 84.94 & $0-456$ \\
$\quad$ sinais/sintomas (em meses) & & & & & \\
Tempo desde diagnóstico (em & & & & \\
meses) & & & & & \\
\hline
\end{tabular}

Doença de Crohn, em comparação aos participantes com Colite Ulcerosa. Verifica-se, pelos meses indicados tanto no tempo de apresentação de sinais/sintomas como no tempo de diagnóstico, que existem participantes com uma indicação temporal recente (de meses) e outros com uma indicação de anos.

\section{Material}

Para alcançar os objetivos propostos foram aplicados um questionário sociodemográfico e clínico e um conjunto de 97 itens respeitantes a cinco instrumentos de recolha; no total, cada participante respondeu a 108 questões. Todos os itens são de autoavaliação, isto é, a preencher pelo indivíduo. Estimou-se o tempo de preenchimento em cerca de 30 minutos.

O questionário sociodemográfio e clínico inclui questões relativas ao sexo, idade, grau de escolaridade e estado civil; a parte clínica inclui questões sobre o tipo de doença, o tempo de diagnóstico e o tempo de sinais/sintomas.

As variáveis dependentes deste estudo "CMSF" e "CMSM" que, em conjunto, representam a QV - foram acedidas utilizando o Questionário do Estado de Saúde (SF-36) (adaptado para a população portuguesa por Ribeiro (2005). A cotação da escala implica que se proceda à transformação de alguns itens e se utilize uma fórmula que converte a nota num valor entre 0 e 100. Além disso, é possível agrupar as dimensões na CMSF e na CMSM. Tanto em cada dimensão como na forma de componente, quanto mais elevado o valor melhor estado de saúde.

As variáveis "ansiedade" e "depressão" foram acedidas com recurso ao Hospital Anxiety and Depression Scale (HADS). Esta escala, criada por Zigmond e Snaith e traduzida e adaptada para a população portuguesa por Ribeiro, Silva, Ferreira, Martins, Meneses e Baltar (2007), é composta por 14 itens, integrando duas subescalas (uma para cada construto) com 7 itens em cada, sendo que os resultados de cada uma podem variar entre 0 e 21 . A escala apresenta-se, na população portuguesa, como uma escala fiel e com validade convergentedivergente razoável (Ribeiro et al., 2007).

$\mathrm{O}$ instrumento escolhido para avaliar a variável "stress percebido" foi a Escala de Perceção de Stresse de 14 itens (PSS-14) criada por Cohen, Kamarck e Mermelstein e adaptada para a população portuguesa por Ribeiro e Marques. Esta escala contém 14 itens aos quais o sujeito deve responder reportando-se ao modo como se sentiu no último mês. Após a realização da cotação os valores são interpretados por correspondência direta entre nota e nível de stress (quanto mais alta a nota mais alto o nível). Os autores referem uma adequada consistência interna, com a exceção do item 12 que apresenta propriedades métricas muito fracas; deste modo, a escala é administrada sem o referido item 
passando a ser constituída por 13 itens (Ribeiro \& Marques, 2009).

A recolha de dados relativos à variável "alexitimia" foi realizada com recurso à Escala de Alexitimia de Toronto (TAS-20). A versão utilizada, adaptada para a língua portuguesa por Prazeres através da escala criada por Taylor, Bagby e Parker, apresenta, tal como a escala original, uma estrutura de três fatores relacionados com a definição do construto (Prazeres et al., 2000). Este instrumento é constituído por 20 itens aos quais o sujeito deve responder, de acordo com a sua concordância. Após a cotação da escala os valores podem variar entre 20 e 100 , classificando-se o sujeito: a) alexitimico, quando o valor é igual ou superior a $61 ;$ b) não alexitimico, quando o valor é igual ou inferior a 51 ; c) numa zona de fronteira e, por isso, sem classificação, quando o valor se situa entre 52 e 60 (Cruz, 2012). A adaptação portuguesa revela que o nível de escolaridade pode influenciar a compreensão dos itens. Verifica-se consistência interna e precisão teste-reteste adequadas (Prazeres et al., 2000).

A variável "personalidade tipo D" foi avaliada com recurso à Escala de Personalidade Tipo D (DS-14), desenvolvida, traduzida (mas não aferida) para português por Dennolet. Esta escala consiste num questionário com 14 itens, contendo duas subescalas com 7 itens cada: a subescala afetividade negativa e a subescala inibição social. Ambas as escalas são classificadas pelo sujeito de acordo com a sua concordância com cada item com recurso a uma escala do tipo Likert de 5 pontos. Os resultados da cotação podem variar entre 0 e 56, considerando-se a personalidade como sendo do tipo D quando cada uma das subescalas apresenta cotação igual ou superior a 10 (Dennolet, 2005). Verifica-se consistência interna e estabilidade temporal em várias populações (Denollet, 2012).

\section{Procedimento}

O procedimento de recolha de dados foi realizado em colaboração com a Associação Portuguesa da Doença Inflamatória do Intestino (APDI). Após a aprovação do projeto de investigação pela Comissão de Ética da FPCEUP, o questionário foi introduzido na plataforma de preenchimento online LimeSurvey e o link para participação foi disponibilizado pela APDI via email aos seus associados. Os dados recolhidos foram introduzidos e analisados com recurso ao programa informático IBM SPSS Statistics 21.

Os testes estatísticos usados para a análise de resultados foram: medidas descritivas e frequências para caracterizar a amostra ao nível das variáveis em estudo; o coeficiente de correlação momento produto de Pearson e a análise de variância (ANOVA) a um fator para determinar a relação entre as variáveis que compõem o estudo, tanto entre si como com as variáveis dependentes; o teste $\mathrm{t}$ de Student para amostras independentes para comparar diferenças na QV de indivíduos com personalidade tipo $\mathrm{D}$ e não $\mathrm{D}$ e de indivíduos alexitimicos e não alexitimicos; a regressão múltipla hierárquica para estabelecer modelos preditores da QV nos indivíduos com DII. No Estudo 1 foram ainda utilizadas a análise fatorial exploratória, solução varimax e o alfa de Cronbach.

\section{Resultados e Discussão do Estudo 1}

Dada a não existência de valores do último instrumento referentes à população portuguesa, procedeu-se à reprodução das principais análises do estudo original (Denollet, 2005), análise fatorial exploratória da escala na amostra do estudo com o objetivo de avaliar características psicométricas do instrumento para a população portuguesa em pessoas com DII.

Utilizou-se a análise de componentes principais por rotação varimax, com valor próprio superior a 1. Foi também calculado o índice de fiabilidade pelo alfa de Cronbach. Os dados obtidos demonstram que a dimensionalidade da escala é consistente com o modelo teórico da personalidade tipo $\mathrm{D}$; a análise da Matriz de Componente Rotativa (Quadro 2) demonstra a existência de dois componentes, coincidentes com as subescalas determinadas pelo instrumento original, tendo os mesmos itens saturados os mesmos fatores, com cargas fatoriais superiores a .500. A escala apresenta elevada consistência interna, no que diz respeito a cada uma das subescalas Afetividade Negativa (.888) e Inibição Social (.871) valores muito semelhantes aos encontrados por Denollet na escala original, tendo sido, respectivamente (.88 e .86). Face aos bons 
Quadro 2. Matriz de Componente Rotativa da escala DS-14

\begin{tabular}{|c|c|c|c|c|}
\hline & \multicolumn{4}{|c|}{ Componente } \\
\hline & & & & \\
\hline & Presente estudo & Estudo original & Presente estudo & Estudo original \\
\hline Item 1 & .053 & -.05 & .799 & -.82 \\
\hline Item 2 & 672 & .69 & .098 & .07 \\
\hline Item 3 & -.126 & .05 & .538 & -.68 \\
\hline Item 4 & .828 & .74 & .152 & .17 \\
\hline Item 5 & .784 & .70 & .118 & .07 \\
\hline Item 6 & .455 & .34 & .676 & .70 \\
\hline Item 7 & 695 & .78 & 179 & .22 \\
\hline Item 8 & .151 & .16 & .847 & .79 \\
\hline Item 9 & .710 & .72 & 227 & .15 \\
\hline Item 10 & .255 & .14 & .801 & .74 \\
\hline Item 11 & 342 & .24 & .692 & .62 \\
\hline Item 12 & .808 & .75 & .020 & .10 \\
\hline Item 13 & .810 & .82 & 184 & .17 \\
\hline Item 14 & .233 & .17 & .723 & .74 \\
\hline
\end{tabular}

resultados de avaliação preliminar psicométrica do instrumento obtida prosseguiu-se para o estudo 2.

\section{Resultados do Estudo 2}

Caraterização da amostra ao nível das variáveis psicológicas em estudo

Para caracterizar as variáveis psicológicas nas quais a amostra $(N=208)$ foi avaliada recorreu-se aos valores da média e do desvio padrão. Esta análise demonstra que a média da CMSF 63.99 (20.73) é superior à média da CMSM 58.17 (23.32); assim, verifica-se que a QV se encontra mais afetada na componente mental do que na física. Além disso, fornece informações relativamente às variáveis independentes: a média da ansiedade 9.09 (3.55) indica a pertença ao grupo ansiedade ligeira e a média da depressão 5.27 (3.95) revela que os valores são normais; a média do stress percebido 25.59 (6.83) indica que o valor é cerca de metade do máximo disponibilizado pela escala e a média da alexitimia 53.65 (12.92) coloca o valor numa zona sem classificação. A prevalência da Personalidade tipo $\mathrm{D}$ foi de $48.6 \%$ ligeiramente inferior à Personalidade tipo Não D 51.4\%. A prevalência da aleximia no estudo foi de $33.17 \%$.

Relação das variáveis independentes com as variáveis dependentes

Recorrendo ao Coeficiente de Correlação momento-produto de Pearson (Quadro 3) verificase que as variáveis dependentes CMSF e CMSM têm uma correlação positiva forte. Por outro lado, as correlações das variáveis independentes psicológicas com as variáveis dependentes são negativas sendo estas correlações mais fortes com a CMSM do que com a CMSF. No caso da CMSM a maioria das correlações são fortes, com a exceção da correlação da alexitimia que é média; a correlação mais forte é com a depressão. No caso da CMSF as correlações não são, de um modo geral, tão elevadas: a maioria das correlações é média sendo as da alexitimia e personalidade tipo D fracas; a correlação mais forte é com a depressão.

Já no que diz respeito à correlação das variáveis independentes sociodemográficas e clínicas (Quadro 3) verifica-se que não existem correlações significativas com a CMSF. Em relação à CMSM apenas as variáveis idade, tempo desde início de sinais e sintomas e tempo desde o

Quadro 3. Correlações das variáveis dependentes entre si e com as variáveis independentes

\begin{tabular}{lcc}
\hline & CMSF & CMSM \\
\hline CMSF & $.714^{* *}$ & $.714^{* *}$ \\
CMSM & $-.508^{* *}$ & $-.700^{* *}$ \\
Ansiedade & $-.531^{* *}$ & $-.717^{* *}$ \\
Depressão & $-.448^{* *}$ & $-.695^{* *}$ \\
Stress percebido & .032 & $.163^{*}$ \\
Idade & -.019 & .092 \\
Diagnóstico & .036 & $.147^{*}$ \\
Tempo desde início & & \\
de sinais e sintomas & & $.137^{*}$ \\
Tempo desde o & .039 & \\
diagnóstico & & \\
\hline
\end{tabular}

** Correlação significativa $p<.01$

* Correlação significativa $p<.05$ 
Quadro 4. Correlações das variáveis independentes psicológicas entre si

\begin{tabular}{lccccc}
\hline & Ansiedade & Depressão & $\begin{array}{c}\text { Stress } \\
\text { percebido }\end{array}$ & Alexitimia & $\begin{array}{c}\text { Personalidade } \\
\text { tipo D }\end{array}$ \\
\hline Ansiedade & & $.70^{* *}$ & $.73^{* *}$ & $.55^{* *}$ & $.61^{* *}$ \\
Depressão & $.70^{* *}$ & & $.74^{* *}$ & $.53^{* *}$ & $.63^{* *}$ \\
Stress percebido & $.73^{* *}$ & $.74^{* *}$ & & $.53^{* *}$ & $.59^{* *}$ \\
Alexitimia & $.55^{* *}$ & $.54^{* *}$ & $.53^{* *}$ & & $.63^{* *}$ \\
Personalidade tipo D & $.61^{* *}$ & $.63^{* *}$ & $.59^{* *}$ & $.63^{* *}$ & \\
\hline
\end{tabular}

**. Correlação significativa $p<.01$

Quadro 5. Comparação de resultados entre participantes, de acordo com o tipo de doença, relativamente às variáveis em estudo

\begin{tabular}{lccccccc}
\hline & \multicolumn{2}{c}{ Doença de Crohn } & \multicolumn{3}{c}{ Colite Ulcerosa } & \multicolumn{3}{c}{ t de Student } \\
\cline { 2 - 8 } & $M$ & $D P$ & $M$ & $D P$ & $t$ & $d f$ & $p$ \\
\hline CMSF & 63.67 & 21.78 & 64.48 & 19.13 & -.28 & 206 & .783 \\
CMSM & 59.91 & 22.37 & 55.51 & 24.62 & 1.33 & 206 & .185 \\
Ansiedade & 8.67 & 3.31 & 9.73 & 3.83 & -2.12 & 206 & .035 \\
Depressão & 4.95 & 3.85 & 5.76 & 4.08 & -1.44 & 206 & .152 \\
Stress percebido & 24.78 & 6.39 & 26.83 & 7.31 & -2.14 & 206 & .034 \\
Alexitimia & 53.32 & 12.89 & 54.17 & 13.05 & -.46 & 206 & .643 \\
Personalidade tipo D & 25.08 & 11.63 & 27.73 & 12.99 & -1.54 & 206 & .126 \\
Idade & 37.31 & 10.28 & 35.74 & 9.57 & 1.103 & 20 & .271 \\
$\begin{array}{l}\text { Tempo desde início de } \\
\text { sinais e sintomas }\end{array}$ & 130.41 & 104.03 & 86.00 & 65.74 & 3.366 & 206 & .001 \\
$\begin{array}{l}\text { Tempo desde } \\
\text { diagnóstico }\end{array}$ & 111.01 & 93.86 & 82.54 & 65.91 & 2.389 & 206 & .018 \\
\hline
\end{tabular}

diagnóstico se correlacionam significativamente, sendo estas correlações positivas e fracas. Recorrendo ao teste $\mathrm{t}$ de Student para amostras independentes verifica-se que não existem diferenças em função do sexo para a CMSF $(t(206)=1.878 ; p=.062)$, nem para a CMSM $(t(206)=1.967 ; \quad p=.051)$. O estado civil foi dicotomizado incluindo (1) solteiros, divorciados e viúvos e (2) casados; também não se verificam diferenças em função desta variável para a CMSF $(t(206)=.240 ; p=.810)$ nem para a CMSM $(t(206)=1.535 ; p=.126)$.

Influência das variáveis personalidade tipo $D e$ alexitimia na $Q V$

Para compreender a influência da variável independente "personalidade tipo D" a amostra foi dividida de acordo com os indivíduos terem ou não personalidade tipo $\mathrm{D}$. Com recurso ao teste $\mathrm{t}$ de Student para amostras independentes é possível concluir que existem diferenças significativas na QV dos pacientes em função da personalidade na CMSM $(t(206)=3.94 ; p=.000)$ sendo que a média da QV é inferior no grupo com personalidade do tipo D. Na CMSF não são encontradas diferenças significativas $(t(206)=1.79 ; p=.075)$.

A amostra foi também dividida em dois grupos: participantes alexitimicos $(N=69)$ e participantes não alexitimicos (incluindo também neste grupo os participantes em zona sem classificação) $(N=139)$. Com recurso ao teste $\mathrm{t}$ de student para amostras independentes conclui-se que há diferenças significativas na QV dos pacientes em função de serem ou não alexitimicos, tanto na $\operatorname{CMSF}(t(206)=2.77$; $p=.006)$. como na CMSM $(t(206)=5.45 ; p=.000)$. sendo a média da QV inferior no grupo alexitimico. Além disso, as médias demonstram que esta diferença é mais acentuada ao nível da CMSM.

\section{Relação das variáveis independentes entre si}

Utilizando o coeficiente de correlação momento-produto de Pearson nas variáveis independentes (Quadro 4) é possível concluir que todas se associam de modo positivo e forte. Reportando-nos às correlações mais fortes verificamos que tanto na ansiedade como na 
Quadro 6. Sumário, ANOVA e correlações do modelo de regressão múltipla hierárquica que prevê a CMSF

\begin{tabular}{|c|c|c|c|c|}
\hline \multirow{2}{*}{$\begin{array}{l}\text { Mode } \\
1\end{array}$} & \multicolumn{2}{|c|}{ R Quadrado } & $\begin{array}{l}\text { Alteração de R } \\
\text { Quadrado }\end{array}$ & $p$ \\
\hline & \multicolumn{2}{|c|}{.036} & .036 & .006 \\
\hline \multirow[t]{2}{*}{2} & \multicolumn{2}{|c|}{.324} & .289 & .000 \\
\hline & & $d f$ & $F$ & $p$ \\
\hline 2 & $\begin{array}{c}\text { Regressão } \\
\text { Residual }\end{array}$ & $\begin{array}{c}4 \\
203\end{array}$ & 24.38 & .000 \\
\hline 2 & $\begin{array}{r}\text { Alexitimia (não } \\
\text { A } \\
\text { Stres }\end{array}$ & vs. ser) & $\begin{array}{c}\beta \\
.088 \\
-.292 \\
-.362 \\
-.001\end{array}$ & $\begin{array}{c}p \\
.180 \\
.002 \\
.000 \\
.993\end{array}$ \\
\hline
\end{tabular}

Quadro 7. Sumário, ANOVA e correlações do modelo de regressão múltipla hierárquica que prevê a CMSM

\begin{tabular}{|c|c|c|c|c|}
\hline Model & R Quadrado & & Alteração de R Quadrado & $p$ \\
\hline 1 & .156 & & .156 & .000 \\
\hline 2 & 636 & & .480 & .000 \\
\hline & & $d f$ & $F$ & $p$ \\
\hline 2 & Regressão & 8 & 43.53 & .000 \\
\hline & Residual & 199 & & \\
\hline & & & $\beta$ & $p$ \\
\hline & Alexitimia (não ser alexitimi & & .015 & .762 \\
\hline & Personalidade tipo $\mathrm{D}$ (personalidade ti & tipo D) & .077 & .110 \\
\hline & Idade & & .123 & .010 \\
\hline 2 & Tempo desde o início dos sina & & -.183 & .099 \\
\hline & Tempo desde o diagnó & & .202 & .064 \\
\hline & Ansiedade & & -.298 & .000 \\
\hline & Depressão & & -.396 & .000 \\
\hline & Stress percebido & & -.215 & .003 \\
\hline
\end{tabular}

depressão é com o stress percebido, no stress percebido com a depressão, na alexitimia com a personalidade tipo D e na personalidade tipo D com a depressão e a alexitimia.

\section{Influência das variáveis em estudo de acordo com} o tipo de doença

Para comparar diferenças nas variáveis de acordo com o tipo de doença foi utilizado o teste $\mathrm{t}$ de Student para amostras independentes (Quadro 5). Os resultados indicam que nas variáveis ansiedade e stress percebido há diferenças significativas entre os dois grupos de participantes; ambas as variáveis têm valores superiores no grupo da $\mathrm{CU}$ do que no grupo da DC. Foram também consideradas variáveis sociodemográficas e clínicas, com o objetivo de procurar explicar as diferenças encontradas. São encontradas diferenças significativas ao nível do tempo desde início de sinais e sintomas e do tempo desde o diagnóstico; as médias demonstram maior precocidade ao nível destes valores na DC.

Estabelecimento de um modelo preditor para a $Q V$

Através da utilização do teste de regressão múltipla hierárquica, usando o método enter, procurou-se compreender o papel de cada uma das variáveis estudadas na predição da QV. Nesta análise foram utilizadas as variáveis independentes psicológicas, sociodemográficas e clínicas, sendo as variáveis alexitimia e personalidade tipo $\mathrm{D}$ consideradas enquanto variáveis dicotómicas. Para a determinação das variáveis incluídas na regressão foram considerados os valores de associação com a QV previamente obtidos. Foi também testada a multicolinearidade para a ansiedade, depressão e stress através do VIF (Variance inflation factor) nos modelos preditores da componente medida sumária física e mental. 
Predição da Componente Medida Sumária Física

Os valores de VIF evidenciados pela ansiedade, depressão e stress foram respetivamente $(2.507 ; 2.578 ; 2.769)$ e atestam que não houve multicolinearidade. Na regressão realizada, a CMSF da QV foi tratada como variável dependente e as restantes variáveis consideradas como tendo influência significativa (alexitimia, ansiedade, depressão e stress percebido) como independentes. A variável alexitimia (não ser alexitimico vs. ser) foi controlada estatisticamente no modelo 1 . Utilizando apenas esta variável o modelo explica $3.6 \%$ da variância; contudo, aquando da introdução das restantes variáveis o modelo explica $32.4 \%, F(4,203)=24.38, p=.000$. No modelo final (Quadro 6), apenas a ansiedade e a depressão têm uma contribuição significativa sendo a da depressão mais elevada do que a da ansiedade.

\section{Predição da Componente Medida Sumária Mental}

Os valores de VIF apresentados pela ansiedade, depressão e stress foram respetivamente $(2.590 ; 2.717 ; 2.795)$ o que comprova que não houve multicolinearidade. $\mathrm{Na}$ regressão realizada, a CMSM da QV foi tratada como variável dependente e as restantes variáveis consideradas como tendo influência significativa (idade, tempo desde início de sinais e sintomas, tempo desde diagnóstico, alexitimia, ansiedade, depressão, personalidade tipo $\mathrm{D}$ e stress percebido) como independentes. As variáveis alexitimia (não ser alexitimico vs. ser) e personalidade tipo $\mathrm{D}$ (personalidade tipo não $\mathrm{D}$ vs. tipo D) foram controladas estatisticamente no modelo 1. Utilizando apenas estas variáveis o modelo explica $15.6 \%$ da variância; contudo, aquando da introdução das restantes variáveis o modelo explica $63.6 \%, F(8,199)=43.53$, $p=.0007$. No modelo final (Quadro 7), apenas a depressão, a ansiedade, o stress percebido e a idade têm uma contribuição significativa apresentando-se aqui as variáveis por ordem decrescente de contributo.

\section{Discussão dos Resultados do Estudo 2}

A presente investigação propõe-se caracterizar num primeiro momento, a $\mathrm{QV}$ na população com
DII quando em comparação com populações sem doença. Constatamos que os valores obtidos na CMSF e na CMSM são inferiores aos obtidos nas mesmas variáveis quando avaliadas numa população saudável (Tudoran \& Furtunescu, 2013; Ribeiro, 2005).

Além disso, se compararmos os valores obtidos na presente amostra concluímos que a CMSM se encontra mais afetada do que a CMSF. Podemos interpretar esta informação considerando que a DII, quando num estado inativo, não provoca sintomas físicos nem limita a atividade física do paciente. No entanto, os valores obtidos no que diz respeito à CMSM levam a hipótese que as exigências psicológicas se mantêm mesmo num estado inativo.

A investigação ao nível da componente psicológica associada à DII na população portuguesa é ainda reduzida. No entanto, existem já estudos que englobam as variáveis ansiedade, depressão, stress e alexitimia que permitem analisar os resultados encontrados à luz da população portuguesa.

As médias encontradas no presente estudo para a ansiedade e depressão, colocam a ansiedade num nível ligeiro e a depressão num nível normal. Por outro lado, se em comparação com amostras saudáveis (Goodhand et al., 2012; Ribeiro et al., 2007), verificamos que tanto os valores da ansiedade como os da depressão são superiores na presente amostra. Assim, estas comparações estabelecidas com amostras saudáveis de outros estudos permitem constatar que a ansiedade e a depressão se encontram num nível superior na população com DII.

Verificamos que o valor da ansiedade é superior ao da depressão. Esta informação é consistente com resultados já encontrados noutros estudos uma vez que, tal como Sajadinejad et al. (2012a) referem, a prevalência superior da ansiedade quando em comparação à da depressão na DII já foi relatada.

No caso da ansiedade verifica-se, ainda, que existe uma diferença significativa no seu nível quando comparando pacientes com CU e com DC, sendo o valor da variável superior nos primeiros. Estes valores vão contra os referidos no estudo de Nordin et al. (2002) que indicam a ansiedade como sendo mais elevada na DC. Procurando explicações para esta diferença verificamos que 
Goodhand et al. (2012) referem como fatores associados à ansiedade na $\mathrm{CU}$ um novo diagnóstico de DII e o stress psicológico. De facto, as variáveis tempo desde o início de sinais e sintomas, tempo desde o diagnóstico e stress percebido foram as únicas, além da ansiedade, a apresentarem diferenças significativas quando comparamos os dois grupos. No grupo com CU esses tempos são inferiores aos relatados pelo grupo com DC. Seguindo esta linha de raciocínio podemos sugerir que mais tempo de vivência da doença contribui para uma melhor adaptação à mesma e para a aquisição de estratégias de coping mais adaptativas. Do mesmo modo, verificamos que os relatos de stress são superiores no grupo com CU; os resultados demonstram, também, uma forte correlação entre as duas variáveis, sendo essa associação também relatada por Ribeiro e Marques (2009). Assim, podemos sugerir que a perceção superior de stress influencia o relato de ansiedade de modo a aumentá-lo.

Ainda no que diz respeito à perceção do stress, verificamos que este é relatado de modo superior numa amostra clínica quando em comparação com uma saudável (Ribeiro \& Marques, 2009; Kuroki et al., 2011). Assim, podemos concluir que associado à vivência da DII o indivíduo perceciona mais stress.

No que diz respeito à alexitimia verifica-se que o valor médio obtido nesta amostra situa-se numa zona de fronteira e, portanto, sem classificação. Estas considerações ganham especial relevância se compararmos a população clínica com DII do presente estudo com populações normativas. Nas populações de Mattila et al. (2009) verifica-se que a média obtida relativamente à alexitimia classifica os participantes saudáveis como "não alexitimicos".

Uma das explicações possíveis para o nível de alexitimia encontrado relaciona-se com as características sociodemográficas da amostra. Como referido, a alexitimia correlaciona-se, entre outros aspetos, com a idade mais avançada, o sexo masculino, baixa escolaridade e estado civil solteiro (Lane, Sechrest, \& Riedel, 1998; Mattila et al., 2009). A presente amostra tem uma idade média próxima dos 40 anos, há predominância de participantes do sexo feminino, de participantes com ensino superior e de participantes casados.
Ao contrário das variáveis referidas, a personalidade tipo $\mathrm{D}$ é uma variável pouco estudada. No presente estudo verifica-se que quase metade da amostra foi avaliada como tendo personalidade tipo D. Em comparação com uma população saudável (Sajadinejad et al., 2012b; Williams, Curren, \& Bruce, 2011; Polman, Borkoles, \& Nicholls, 2010; Denollet, 2005) verifica-se que a prevalência é mais elevada. Por outro lado, comparando com populações clínicas com outras doenças (Sararoudi et al., 2011; Denollet, 2005) a prevalência aproxima-se sendo, no entanto, quase sempre superior na presente amostra.

Ainda que as informações relativas a esta variável noutros estudos sejam escassas podemos concluir que a sua incidência se demonstra aumentada nas populações clínicas e que a sua prevalência estará relacionada com o tipo de doença. Esta prevalência poderá, também, encontrar-se relacionada com a presença de outras variáveis psicológicas. De facto, as correlações encontradas entre as variáveis do presente estudo parecem corroborar esta hipótese e vão de encontro ao exposto na introdução teórica: este tipo de personalidade como associado ao stress percebido (Polman, Borkoles, \& Nicholls, 2010), à depressão e ansiedade (Denollet, 2005) e à alexitimia (Williams, Curren, \& Bruce, 2011).

Considerando os resultados discutidos até ao momento verificamos que os valores de ansiedade, depressão e stress percebido são superiores aos encontrados em populações saudáveis supramencionadas. Do mesmo modo, embora os valores da alexitimia coloquem os participantes numa zona fronteira, que careceria de mais investigação, nas populações saudáveis referidas os participantes eram considerados não alexitimicos. No que diz respeito à personalidade tipo $\mathrm{D}$ verifica-se que, em comparação com populações saudáveis, a sua prevalência é mais elevada.

Tendo em conta estas conclusões existem dois aspetos a ter em conta: o modo como as referidas variáveis se correlacionam e o modo como se relacionam com a $\mathrm{QV}$.

A ansiedade, depressão, stress percebido, alexitimia e personalidade tipo D apresentam, entre si, associações significativas e positivas. Como demonstrado na introdução teórica, 
diversas das associações entre as referidas variáveis são demonstradas pela literatura. Além deste conhecimento, as correlações obtidas demonstram que níveis mais elevados de ansiedade, depressão e stress percebido se relacionam com níveis mais reduzidos de $\mathrm{QV}$; tal como apresentado, esta conclusão vai de encontro à literatura.

Como esperado, a influência das variáveis acima referidas é mais notável na CMSM do que na CMSF. Verificamos que a depressão é a variável com uma correlação mais forte com ambas as componentes, que a ansiedade e o stress percebido têm a mesma correlação com a CMSM mas a ansiedade tem uma correlação mais forte com a CMSF. Esta influência considerável pode ser parte da explicação para o facto destas variáveis serem mais estudadas na literatura quando em comparação com a alexitimia e a personalidade tipo $\mathrm{D}$.

A análise efetuada para avaliar a influência da alexitimia e da personalidade tipo D na $\mathrm{QV}$ foi diferente, tendo em conta a estrutura dos instrumentos utilizados na sua avaliação e, também, o facto de serem as variáveis menos estudadas. No entanto, também esta análise permite verificar que a alexitimia e a personalidade tipo D têm influência na QV.

Como referido na introdução teórica, já vários estudos demonstraram a relação entre alexitimia e QV. Os resultados obtidos neste estudo demonstram que há uma diferença significativa nos relatos de $\mathrm{QV}$, em ambas as componentes, sendo ou não alexitimico. Os participantes avaliados como alexitimicos têm uma QV inferior à dos participantes avaliados como não alexitimicos, sendo esta diferença mais acentuada ao nível da CMSM.

Encontramos resultados semelhantes no que diz respeito à personalidade tipo $\mathrm{D}$, embora apenas na CMSM; comparando as médias dos dois grupos verificamos que os participantes com personalidade tipo não D têm uma QV na CMSM superior à dos participantes com personalidade tipo D. Podemos então verificar uma influência da personalidade tipo $\mathrm{D}$ na $\mathrm{QV}$. Ainda que a literatura nesta temática seja mais reduzida, utilizando estudos com populações semelhantes como comparação, os resultados encontrados vão no sentido esperado.
Embora até ao momento tenha sido referida apenas a influência das variáveis psicológicas na $\mathrm{QV}$, é também de considerar a influência das variáveis sociodemográficas e clínicas. Verificamos que a idade, o tempo desde o início de sinais e sintomas e o tempo desde o diagnóstico se correlacionam com a CMSM. Os resultados encontrados permitem concluir que aumento da idade, bem como um maior tempo de convivência com a doença, se associam a um relato da QV na CMSM mais positivo.

Embora o aumento da idade seja indicado por Sainsbury e Heatley (2005) como tendo influência negativa na $\mathrm{QV}$, os autores também referem na sua revisão um estudo grego que indica que a única diferença relevante associada à idade em pacientes com mais de 60 anos era o reduzido funcionamento social. Assim, são de realçar, além da idade média da amostra, a predominância do estado civil casado e a pertença à APDI pelo que a questão do funcionamento social pode perder a importância.

Por outro lado, ao aumento da idade podem estar associadas estratégias de coping mais adaptativas. De facto, o estudo de Diehl, Coyle e Labouvie-Vief (1996), relacionado com a modificação das estratégias de coping ao longo da vida, sugere que com o aumento da idade pode haver um aprimorar das estratégias de coping. É também de voltar a referir a influência do tempo de convivência com a doença e que pode, também aqui, ajudar a explicar a influência positiva nos relatos de QV: mais tempo de doença pode estar relacionado com mais tempo para a adoção de estratégias de coping mais adaptativas.

Considerando estas conclusões mostrou-se importante compreender quais as variáveis que permitem a predição da QV. Verificamos que a ansiedade e a depressão são as únicas variáveis introduzidas que desempenham um papel na QV, em ambas as componentes. De facto, a literatura defende que estas podem influenciar a perceção dos sintomas físicos e, assim sendo, afetar a descrição feita da QV nessa componente (Hauser et al., 2011). Ainda nas variáveis psicológicas que desempenham um papel relevante encontramos o stress percebido (ainda que apenas na CMSM). Tendo em conta os dados que indicam uma estreita relação entre stress, ansiedade e depressão (Ribeiro \& Marques, 2009) é compreensível que 
as três variáveis desempenhem este papel na predição da CMSM. Das variáveis sociodemográficas, apenas a idade se apresenta como significativa na CMSM da QV não sendo o seu papel tão significativo como o da depressão, ansiedade e stress percebido. Esta influência da idade já foi explicada, acima, na presente discussão.

A criação de tais modelos preditores é útil na medida que permite verificar se variáveis que demonstram uma associação significativa em termos estatísticos com a QV mantém a sua associação quando avaliadas em conjunto com outras variáveis. Tal não significa que variáveis que não integram os modelos preditores não possam exercer a sua influência mas é um indicador de quais as variáveis que, na prática clínica, devem ser alvo de especial atenção.

\section{Conclusão}

A população com DII não é frequentemente estudada, sobretudo envolvendo esta população em Portugal. Deste modo, a presente investigação permite aprofundar o conhecimento pré-existente, fazendo-o através de uma amostra superior àquela que geralmente figura neste tipo de investigações. $\mathrm{O}$ aumento informacional relativo aos fatores que podem impactar a QV permite aos profissionais de saúde, nomeadamente aos psicólogos, atuar de modo mais antecipado e mais eficaz.

Um dos aspetos inovadores deste estudo é o interesse pelo impacto da alexitimia e da personalidade tipo $\mathrm{D}$ na $\mathrm{QV}$ e nesta população; ainda que as variáveis ansiedade, depressão e stress percebido sejam alvo frequente de estudo, nesta e noutras populações, o mesmo não é verdade quanto às primeiras. Salienta-se que a personalidade tipo D é um constructo recente e pouco estudado mesmo a nível internacional, pelo que incluir esta variável no estudo o torna particularmente relevante. Neste sentido, a presente investigação permitiu verificar que a Escala de Personalidade Tipo $\mathrm{D}$, embora não aferida para a população portuguesa, apresenta, numa amostra portuguesa com DII, subescalas coincidentes com as do instrumento original bem como valores de elevada consistência interna. Seria importante, no futuro, realizar o mesmo tipo de aferição para uma população saudável que permitisse comparações mais exactas.

A nível de intervenção psicológica, para melhorar sobretudo a CMSM constatamos a importância sobre a regulação emocional nesta patologia no que reporta a falta de expressividade emocional detetada pela prevalência de alexitimia a acrescer às perturbações de humor e predomínio de inibição social e afetividade negativa na personalidade tipo $\mathrm{D}$, sugerindo o estimular das redes de suporte social destes pacientes.

Uma vez que a recolha de dados foi transversal o presente estudo não permite que sejam retiradas conclusões acerca da influência do processo de adaptação à doença por parte dos participantes. Do mesmo modo, não permite aferir se as variáveis psicológicas analisadas se encontravam já presentes aquando do diagnóstico ou se surgem em consequência do mesmo. Além disso, a não existência de uma amostra de controlo não permite a comparação com uma população normativa que tenha sido avaliada exatamente do mesmo modo que a amostra clínica.

No que diz respeito a investigações futuras, seria interessante realizar o estudo numa amostra que não pertença a uma associação e que, assim, não tenha o mesmo tipo de acesso a apoio psicológico podendo ser estabelecidas comparações quanto à influência deste suporte. Além disso, seria interessante avaliar qual o impacto da fase de adaptação à doença na qual os participantes se encontram. Finalmente, a utilização simultânea de uma metodologia qualitativa permitiria o acesso mais aprofundado à interpretação que os participantes fazem da sua doença e dos aspetos que dificultam a gestão da mesma.

Concluindo, é positivo verificar que ainda que a QV esteja num nível inferior quando em comparação com a população saudável, se situa num nível satisfatório em comparação com outras populações e com o total máximo da escala utilizada para a avaliar. Como já referido, uma hipótese para este fenómeno é o facto de os participantes fazerem parte de uma associação cujas preocupações incluem os aspetos psicológicos. Isto, juntamente com as restantes conclusões do estudo, realça a importância de uma 
abordagem da saúde como constituída pelo bom funcionamento físico, psicológico e social.

\section{Referências}

Anxiety and Depression Association of America (2010-2014). Depression. Anxiety and Depression Association of America (ADAA) Web site. Acedido junho 30, 2014, em http://www.adaa.org/understanding-anxiety/de pression

Anxiety and Depression Association of America (2010-2014). Generalized Anxiety Disorder (GAD). Anxiety and Depression Association of America (ADAA) Web site. Acedido junho 30, 2014, em http://www.adaa.org/understandi ng-anxiety/generalized-anxiety-disorder-gad

Ben Thabet, J., Charfi, N., Mnif, L., Yaich, S., Zouari, L., Zouari, N., et al. (2012). Emotional disorders and inflammatory bowel disease [Abstract]. La Tunisie Medicale, 90(7), 557-563.

Boye, B., Lundin, K. E. A., Leganger, S. Mokleby, K., Jantschek, G., Jantschek, I., et al. (2008). The INSPIRE study: Do personality traits predict general quality of life (Short form-36) in distressed patients with ulcerative colitis and Crohn's disease? Scandinavian Journal of Gastroenterology, 43(12), 1505-1513. doi: 10.1080/0036552080 2321196.

Cruz, I. M. F. (2012). Análise fatorial confirmatória da Escala de Alexitimia de Toronto (TAS-20) numa amostra de indivíduos com anorexia nervosa. Dissertação de Mestrado, Faculdade de Psicologia e de Ciências da Educação - Universidade do Porto, Portugal.

Denollet, J. (2005). DS14: Standard Assessment of Negative Affectivity, Social Inhibition, and Type D Personality. Psychosomatic Medicine, 67(1), 89-97. doi: 10.1097/01.psy.000014925 6.81953.49.

Denollet, J. (2012). Type D or not type D: that's the question. The European Health Psychologist, 14(3), 58-63.

Diehl, M., Coyle, N., \& Labouvie-Vief, G. (1996). Age and sex differences in strategies of coping and defense across the life span. Psychology and Aging, 11(1), 127-139.
Faust, A. H., Halpern, L. F., Danoff-Burg, S., \& Cross, R. K. (2012). Psychosocial factors contributing to inflammatory bowel disease activity and health-related quality of life. Gastroenterology \& Hepatology, 8(3), 173181.

Goodhand, J. R., Wahed, M., Mawdsley, J. E., Farmer, A. D., Aziz, Q., Rampton, D. S. (2012). Mood disorders in inflammatory bowel disease: Relation to diagnosis, disease activity, perceived stress, and other factors. Inflammatory Bowel Diseases, 18(12), 23012309. DOI: 10.1002/ibd.22916.

Hansel, S. L., Umar, S. B., Lunsford, T. N., Harris, L. A., Dibaise, J. K., \& Crowell, M. D. (2010). Personality traits and impaired healthrelated quality of life in patients with functional gastrointestinal disorders. Clinical Gastroenterology and Hepatology, 8(2), 220222. DOI: 10.1016/j.cgh.2009.10.008.

Hauser, G., Tkalcić, M., Stimac, D., Milić, S., \& Sincić, B. M. (2011). Gender related differences in quality of life and affective status in patients with inflammatory bowel disease. Collegium Antropologicum, 35(2), 203-207.

Iglesias, M., Barreiro-de Acosta, M., Vázquez, I., Piñeiro, M., Figueiras, A., Cao, A., et al. (2011). Evaluation of stress in inflammatory bowel disease patients [congress abstract]. European Crohn's and Colitis Organisation (ECCO) Web site. Acedido junho 30, 2014, em https://www.ecco-ibd.eu/publications/co ngress-abstract-s/abstracts-011/item/137.html Iglesias-Rey, M., Barreiro-de Acosta, M., Caamaño-Isorna, F., Rodríguez, I. V., González, A. L., Bello-Paderne, X., et al. (2012). Influence of alexithymia on healthrelated quality of life in inflammatory bowel disease: Are there any related factors? Scandinavian Journal of Gastroenterology, 47(4), 445-453. doi: 10.3109/00365521.2012. 654403.

Iglesias-Rey, M., Barreiro-de Acosta, M., Caamaño-Isorna, F., Rodríguez, I. V., Ferreiro, R., Lindkvist, B., et al. (2014). Psychological factors are associated with changes in the health-related quality of life in inflammatory bowel disease [Abstract]. 
Inflammatory Bowel Disases, 20(1), 92-102. DOI: 10.1097/01.MIB.0000436955.78220.bc.

Kuroki, T., Ohta, A., Sherriff-Tadano, R., Matsuura, E., Takashima, T., Iwakiri, R., et al. (2011). Imbalance in the stress-adaptation system in patients with inflammatory bowel disease. Biological Research for Nursing, 13(4), 391-398. doi: 10.1177/1099800410388 638.

Lane, R. D. Sechrest, L., \& Riedel, R. (1998). Sociodemographic correlates of alexithymia. Comprehensive Psychiatry, 39(6), 377-385.

Lunazzi de Jubany, H. (2000). Investigación de variables en al constructo Alexitimia en 550 casos. Revista Iberoamericana de Diagnóstico y Evaluación - e Avaliação Psicológica, 10(2), 101-119.

Mattila, A. K., Saarni, S. I., Salminen, J. K., Huhtala, H., Sintonen, H., \& Joukamaa, M. (2009). Alexithymia and health-related quality of life in a general population. Psychosomatics, 50(1), 59-68. doi: 10.1176/appi.psy.50.1.59.

Meder, A., Swiatkowaski, M., Meder, G., \& Koza, J. (2010). Quality of life and coexisting anxiety-depression disorders in patients with inflammatory bowel disease during relapse and a further 11 months observation. Gastroenterologia Polska, 17(4), 273-279.

Moradkhani, A., Beckman, L. J., \& Tabibian, J. H. (2013). Health-related quality of life in inflammatory bowel disease: Psychological, clinical, socioeconomic, and demographic predictors. Journal of Crohn's and Colitis, 7(6), 467-473. doi: 10.1016/j.crohns.2012.07. 012.

Nahon, S., Lahmek, P., Durance, C., Olympie, A., Lesgourgues, B., Colombel, J., et al. (2012). Risk factors of anxiety and depression in inflammatory bowel disease. Inflammatory Bowel Diseases, 18(2), 2086-2091. doi: 10.1002/ibd.22888.

Nasseri-Moghaddam, S. (2012). Inflammatory bowel disease. Middle East Journal of Digestive Diseases, 4(2), 77-89.

Nordin, K., Pahlman, L., Larsson, K., SundbergHjelm, M., \& Lööf, L. (2002). Health-related quality of life and psychological distress in a population-based sample of swedish patients with inflammatory bowel disease.
Scandinavian Journal of Gastroenterology, 37(4), 450-457. doi: 10.1080/0036552023173 16097.

Polman, R., Borkoles, E., \& Nicholls, A. R. (2010). Type D personality, stress, and symptoms of burnout: The influence of avoidance coping and social support. British Journal of Health Psycholgy, 15(3), 681-696. DOI: 10.1348/135910709X479069.

Prazeres, N, Parker, D. A., \& Taylor, J. (2000) Adaptação Portuguesa da Escala de Alexitimia de Toronto de 20 Itens (TAS20). Revista Iberoamericana de Diagnóstico y Evaluación - e Avaliação Psicológica, 9(1), 9-21.

Ribeiro, J. P. (2005). O importante é a saúde. Lisboa: Fundação Merck Sharp \& Dohme.

Ribeiro, J. P., \& Marques, T. (2009) A avaliação do stresse: A propósito de um estudo de adaptação da escala de perceção de stresse. Psicologia, Saúde \& Doenças, 10(2), 237248.

Ribeiro, J., Silva, I., Ferreira, T., Martins, A., Meneses, R., \& Baltar, M. (2007). Validation study of a portuguese version of the Hospital Anxiety and Depression Scale. Psychology, Health \& Medicine, 12(2), 225-237.

Sainsbury, A., \& Heatley, R. V. (2005). Review article: Psychosocial factors in the quality of life of patients with inflammatory bowel disease. Alimentary Pharmacology \& Therapeutics, 21(5), 499-508.

Sajadinejad, M. S., Asgari, K., Molavi, H., Kalantari, M., \& Adibi, P. (2012a) Psychological issues in inflammatory bowel disease: An overview. Gastroenterology Research and Practice, 1-11. doi: 10.1155/2012/106502

Sajadinejad, M. S., Molavi, H., Asgari, K., Kalantari, M., \& Adibi, P. (2012b). Personality dimensions and type D personality in female patients with ulcerative colitis. Journal of Research in Medical Sciences, 17(10), 898-904.

Salas, C., \& Garzón, M. O. (2013). La nocíon de calidad de vida y su medición. CES Salud Pública, 4, 36-46.

Sararoudi, R. B., Afshar, H., Adibi, P., Daghaghzadeh, H., Fallah, J., \& Abotalebian, F. (2011). Type D personality and quality of 
life in patients with irritable bowel syndrome. Journal of Research in Medical Sciences, 16(8), 985-992.

Sugrue, K., Landers, M., McCarthy, J., \& Buckley, M. (2013). An investigation of the manifestations of perceived psychological stress in patients with inflammatory bowel disease [congress abstract]. European Crohn's and Colitis Organisation (ECCO) Web site. Acedido junho 30, 2014, em http://www.eccoibd.eu/publications/congress-abstract-s/abstra cts-2013/item/on001-an-investigation-of-themanifestations-of-perceived-psychological-str ess-in-patients-with- inflammatory-bowel-dise ase.html

Todorovic, V. (2012). Providing holistic support for patients with inflammatory bowel disease. British Journal of Community Nursing, 17(10), 466-472.

Tudoran, I., \& Furtunescu, F. L. (2013). Perceived stress and health related quality of life in a group of Romanian adults. AMT, 2(3), 220223.

Williams, L., Curren, C., \& Bruce, G. (2011). Are alexithymia and Type D personality distinct or overlapping constructs? A confirmatory fator analysis of the Toronto alexithymia and Type D scales. Personality and Individual Differences, 51(5), 683-686. doi: 10.1016/j.paid.2011.06.007

Zhou, Y., Ren, W., Irvine, E. J., \& Yang, D. (2010). Assessing health-related quality of life in patients with inflammatory bowel disease in Zhejiang, China. Journal of Clinical Nursing, 19(1-2), 79-88. doiI: 10.1111/j.13652702.2009.03020.x. 